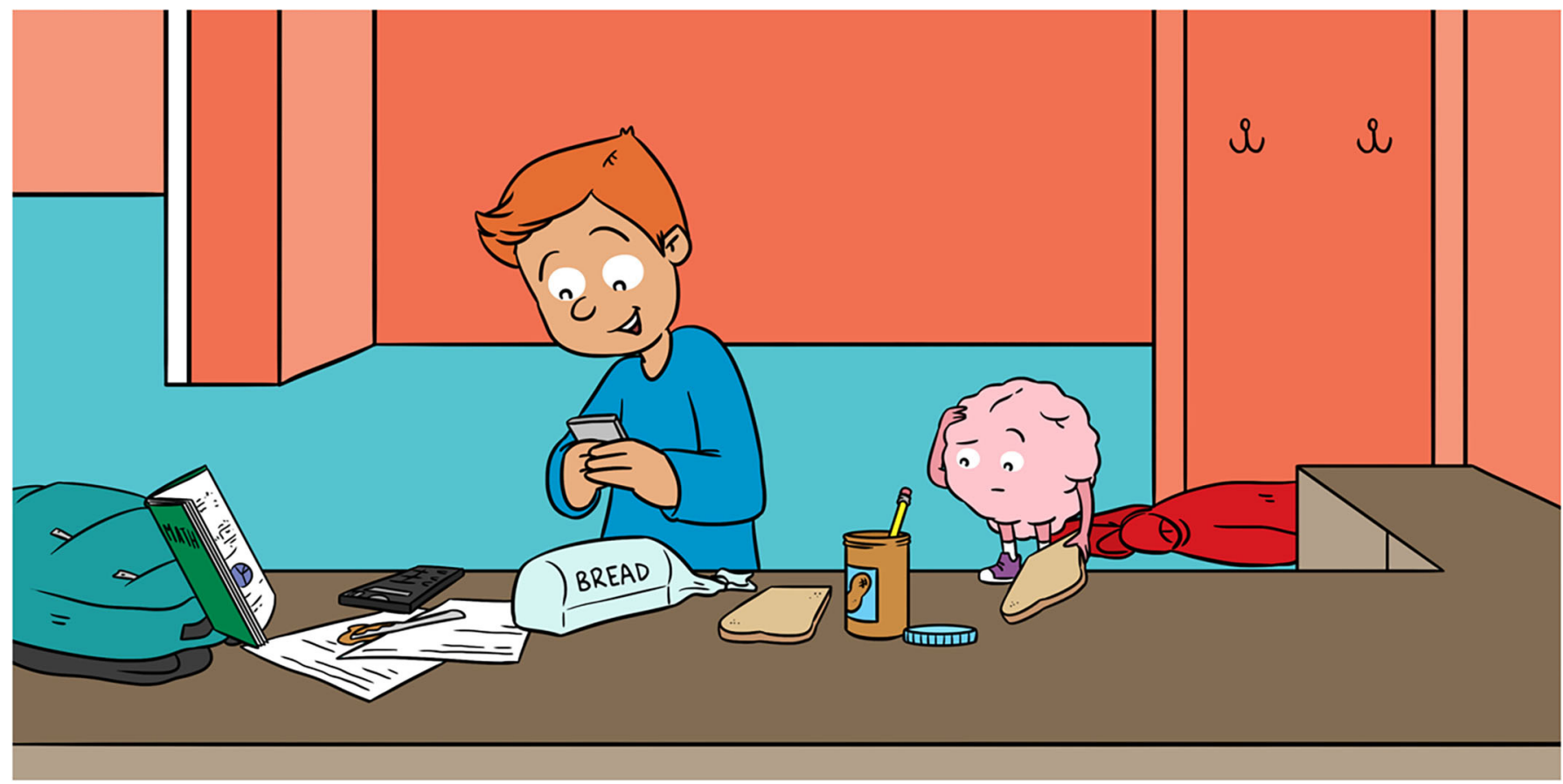

\title{
THE MORE, THE MERRIER? WHAT HAPPENS IN YOUR BRAIN WHEN YOU ARE MULTITASKING?
}

\section{Sina Alexandra Schwarze ", Caroline Poppa ", Sarah Margo Gawronska " and Yana Fandakova * \\ Center for Lifespan Psychology, Max Planck Institute for Human Development, Berlin, Germany}

YOUNG REVIEWER:

TORBEN

AGE: 12
Have you ever thought about what happens in your brain when you engage in multiple tasks at the same time? Although we multitask often throughout the day, limits to our mental capacity prevent our brains from processing multiple tasks truly simultaneously. Instead, we actually switch between them, so rapidly that we may not even be aware of it. Two brain areas appear to be especially important for multitasking - the parietal cortex and the prefrontal cortex, whose complex interaction is an ongoing focus of scientific investigation. With the ever-increasing popularity of new technological devices like smartphones, which tempt us to multitask more frequently, it has become crucial to understand how multitasking affects the ability to complete a task quickly and correctly. While many questions remain about multitasking and its effects on the brain and our cognitive skills, currently available research points to the importance of developing smart multitasking habits. 


\section{COGNITIVE}

Relating to mental processes like learning, thinking, problem-solving, and memory, which are carried out by the brain.

\section{CAN MORE BECOME TOO MUCH?}

Sometimes, more is better. Picture yourself in your kitchen with friends, baking your favorite chocolate chip cookies. You cannot get enough of them, so you have decided to double the recipe today! While gathering the ingredients, your growling stomach reminds you of what you learned in your latest biology lesson, about the fascinating digestive roles of gut bacteria. You begin to share your new knowledge with your friends as you simultaneously try to calculate the doubled measurements and whisk the ingredients together. But wait-how much sugar do you need, exactly? To ensure that you are doing the math correctly, you realize that you need to pause your explanation of gut bacteria. Clearly, more is not always better: while your stomach may be able to handle the extra cookies, adding more tasks to be processed can sometimes prove too much for your brain.

You have likely done two things, such as baking or talking to friends, separately, many times, without any problems. However, if you attempt to do both at once, you might find yourself struggling to handle either task as well as you normally could. This is called the cost of multitasking, and it occurs when the same cognitive (brain) resources are required by two or more tasks at the same time.

Why are brain scientists interested in multitasking? Humans have likely always multitasked, but recently, new technologies have made it a more common feature of our daily activities. Smartphones, for example, have become our constant companions and are often used to do several things simultaneously. Increased multitasking brings up many questions and concerns.

Many parents and teachers worry that the tech-savvy younger generation divides its attention too much between different activities and may grow up to be a bunch of unfocused "scatterbrains." Maybe you have even wondered about the effects of frequent multitasking yourself-is it "good" or "bad" for you? Before we address this question, let us look at what makes multitasking challenging and what happens in the brain.

\section{A BOTTLENECK IN THE BRAIN?}

Why is our ability to perform two tasks at once limited? Scientists have developed laboratory experiments comparing participants' performances when they were performing only one task to when they performed two tasks simultaneously (Figure 1, based on the task described in [1]). In two separate tasks, participants concentrated on a series of single letters. By pressing a button, they had to indicate whether the letter was a consonant or a vowel in the first task, and whether the letter was capitalized or not in the second task. In the so-called single-task condition, participants performed only one task 
Figure 1

Experimental setup. $(A, B)$ In the single-task condition, participants focused on whether the presented letters were written in capital or lowercase, and whether the letters were consonants or vowels. These tasks were performed separately, during independent task sessions. (C) In the dual-task condition participants had to focus on both categories simultaneously for each letter, with $2.5 \mathrm{~s}$ in between letters.

\section{BOTTLENECK}

\section{THEORY}

It states that the brain has a limited capacity for processing multiple tasks simultaneously, just like only one marble can pass through the narrow neck of a bottle at once.

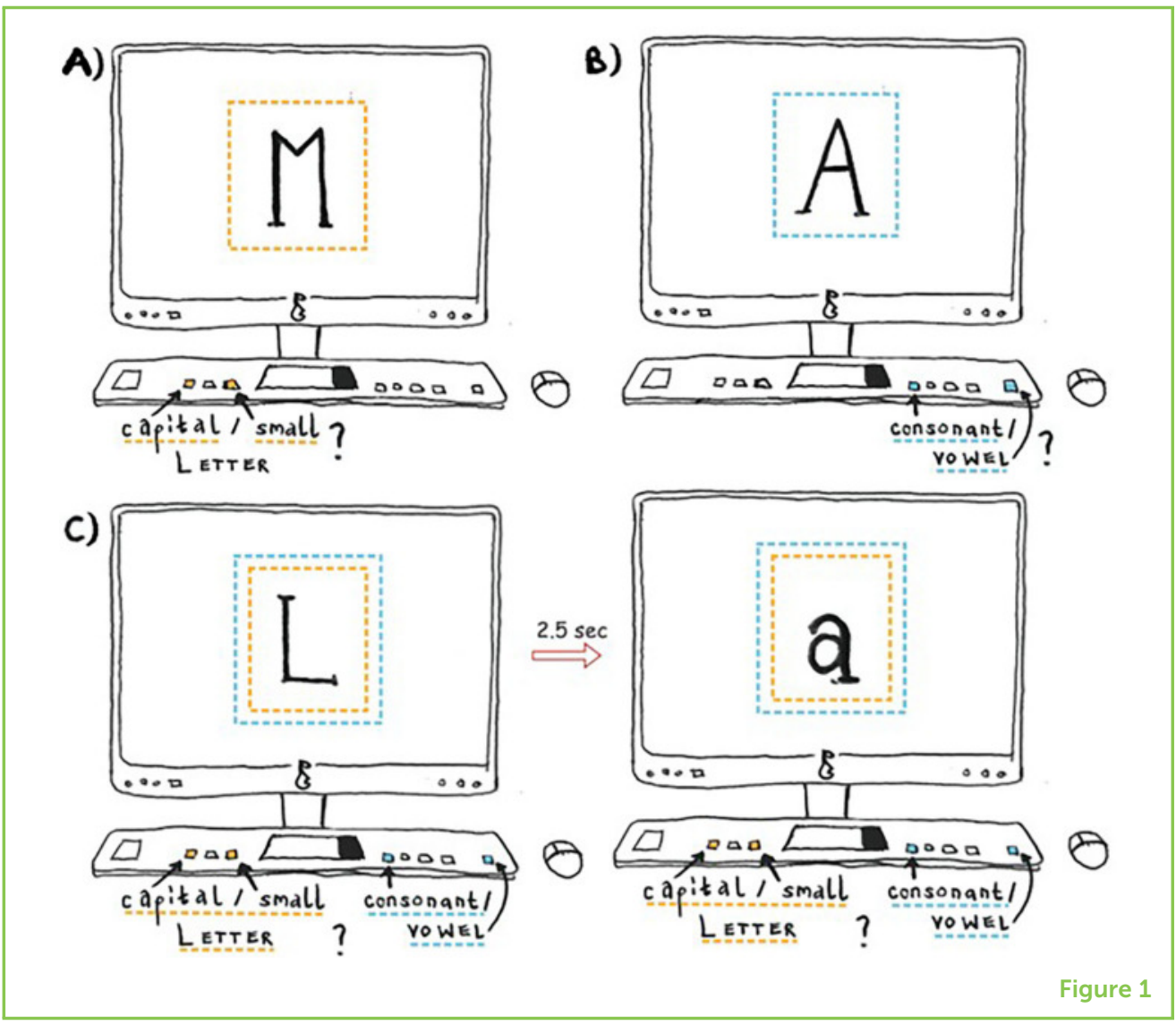

at a time, whereas in the dual-task condition they had to complete both tasks simultaneously. So, while just one button press was required for a response to each letter in the single-task condition, the dual-task condition required two button presses for each letter: one for the vowel/consonant decision and one for the capital letter task. In this experiment and others like it, participants tend to respond more slowly to each of the two tasks in the dual-task condition compared to the single-task condition.

To explain this finding, scientists have developed several theories. One of the most influential is called the bottleneck theory [2]. Think of the neck of a bottle filled with marbles: only one marble at a time can pass through. Similarly, just one task at a time can move through the bottleneck of limited processing capacity in the brain. The other task must wait until the first one is completed.

In our earlier example of baking, measuring sugar and telling your friends about your biology class can be pictured as two separate marbles attempting to move through the bottleneck. Mentally doubling the recipe measurements then adds a third marble that needs to pass. Some of these tasks are more complicated and thus need more of your capacity, represented by the larger size of the marble (Figure 2). The task marbles cannot move through the bottleneck at the same time, so they are dealt with one after another. After partially 


\section{Figure 2}

The bottleneck theory states that our brain has a limited capacity for processing and performing multiple tasks simultaneously. To envision this concept, imagine your brain was a bottle filled with marbles, each marble representing a task. The moment you perform one task, the corresponding marble has to pass through the bottleneck. But, only one marble at a time can pass through the bottleneck.

Similarly, only one task at a time can be processed in your brain. In addition, some tasks require more brain capacity represented by the different sizes of the marbles.

\section{FUNCTIONAL}

\section{MAGNETIC}

\section{RESONANCE}

\section{IMAGING (FMRI)}

fMRI uses a strong magnetic field to take images of the brain. For example, when human participants work on a task, it can show the brain regions that are activated for that task (learn more here: https://kids. frontiersin.org /article/10.3389 /frym.2019.00086).

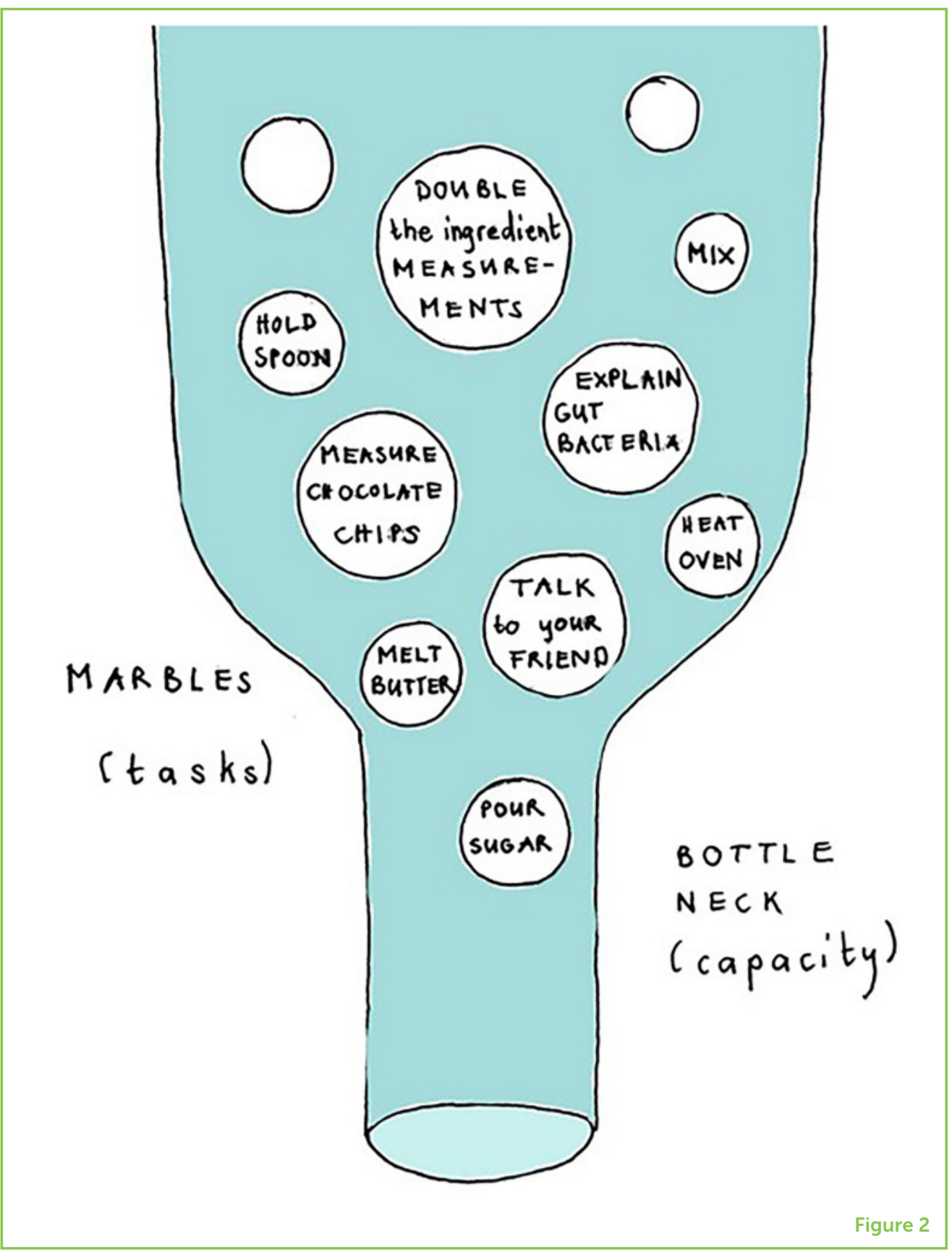

completing one task, you rapidly switch to the next one and then back again to the first, repeatedly. Thus, you feel like you are doing multiple tasks simultaneously.

\section{ARE THERE MULTITASKING "HOTSPOTS" IN THE BRAIN?}

With a technology called fMRI [3], scientists can measure how active the various regions of the brain are while someone performs a task. Using fMRI, scientists have identified two main brain areas where this switch between tasks happens during multitasking. The prefrontal 
Figure 3

Brain regions involved in multitasking. The prefrontal cortex (yellow) and the parietal cortex (green) are both active during multitasking. The parietal cortex stores S-R mappings, that describe the relationship between an external event and the response that is triggered by this event. The prefrontal cortex on the other hand, is essential for selecting the correct $\mathrm{S}$-R-mapping for the task and responding appropriately.

\section{PREFRONTAL}

\section{CORTEX}

A region at the front of the brain, which is involved in multitasking by selecting the correct $\mathrm{S}-\mathrm{R}$ mapping for a task and thus for

responding appropriately.

\section{PARIETAL CORTEX}

A region at the upper back of the brain, which is involved in multitasking by storing the S-R mapping of each task.

\section{STIMULUS}

An event or a cue, such as a picture or a sound, that activates sensory receptors and causes a response from an organism.

\section{STIMULUS-RESPONSE -MAPPING (SHORT: S-R-MAPPING)}

The relation between a stimulus and the response that is appropriate for this stimulus; it can be imagined as a roadmap from stimulus

to response.

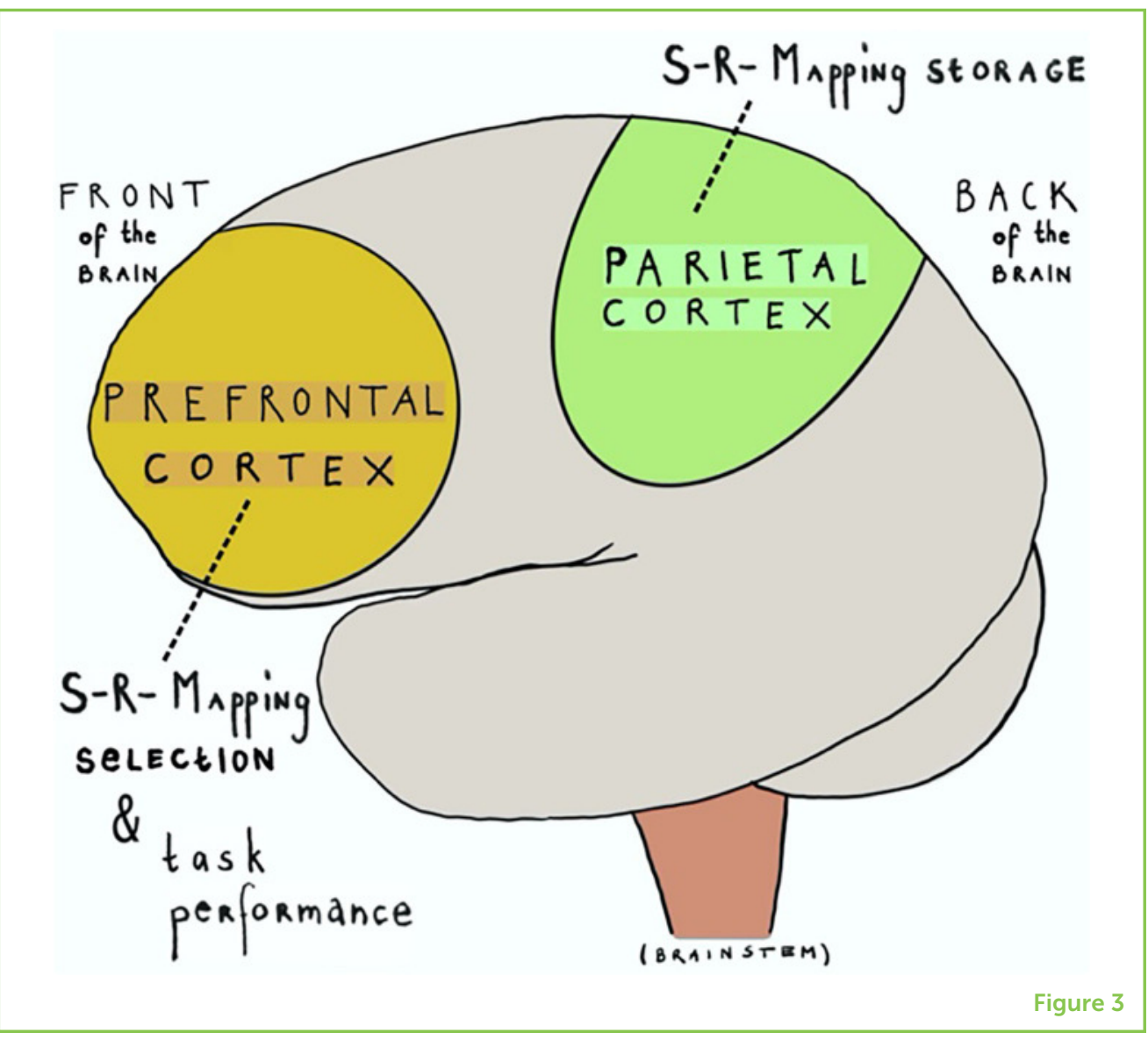

cortex and the parietal cortex are more active when participants complete two tasks at once, compared to when they process a single task. These brain regions thus appear to be essential for multitasking. Using clever experiments, scientists have developed an idea of what these brain areas do when we multitask (Figure 3). The parietal cortex stores the relationship between an external event, such as a picture or a sound (called a stimulus), and the response that should happen because of that stimulus. This is called a stimulus-response-mapping or S-R-mapping. In our example experiment, the task rules of pressing the left yellow button if the letter is capitalized and the right blue button if it is a vowel are examples of S-R-mappings stored in the parietal cortex. The prefrontal cortex is essential for selecting the correct S-R-mappings and responding appropriately. So, if you see a capital " $A$ " in the yellow single-task condition, the prefrontal cortex ensures that you press the left yellow button in response [4]. The prefrontal cortex may be the location of the bottleneck. Practicing multitasking intensively can increase the speed with which the prefrontal cortex processes multiple tasks [5] and can improve multitasking even for tasks that were not practiced, but we still do not know how long-lasting these effects are and whether practice also helps us improve multitasking skills in our daily lives. 
Interestingly, some people can switch between tasks or sources of information more easily than others. While many factors influence the ability to multitask, age is especially important [6]. Perhaps you have observed that your younger siblings have a harder time doing multiple things at a time than you do, or that your own multitasking ability has improved since you were little. That is because the brain regions involved in multitasking and their connections with one another need time to develop, becoming the strongest in late adolescence or early adulthood. Just as your brain keeps changing after adolescence, so does your ability to multitask. As we approach our grandparents' age, the brain regions responsible for task switching function less effectively, making multitasking more difficult again [6].

\section{WE LIVE IN THE AGE OF MEDIA MULTITASKING}

Multitasking can be challenging for people regardless of age, but it is also the "new normal" of our time. Even when the situation does not require it, our electronic devices can tempt us to multitask. You may, for example, enjoy listening to music and keeping an eye on social media while completing your homework. In general, research suggests that media multitasking like this can scatter attention and worsen memory, which may impair learning and performance on a task. Additionally, trying to do multiple things at once does not save more time than doing tasks one after the other-multitasking actually slows us down! This seems to be mostly true even for frequent media multitaskers with much "practice" and confidence in their ability to switch between tasks [7]. However, there may be some exceptions to this rule. Experienced video gamers appear to be able to switch between tasks better than non-gamers, at least if the tasks are not very similar [8]. This does not necessarily mean that video games improve multitasking skills-perhaps people who like to play them are better task switchers to begin with. Finally, researchers are still working to find out if frequent multitasking has any long-term effects, positive or negative, on our cognitive abilities or our brains [6].

\section{BE SMART ABOUT MULTITASKING!}

Although the bottleneck theory has received much support from scientific experiments, there are also competing explanations, and gaps still exist in our knowledge. More research is needed to reveal exactly how multitasking works and why it is challenging to our brains. Multitasking is here to stay, and concerns about its effects will likely persist until more scientific evidence becomes available. In the meantime, it is important to remember that multitasking is a human ability, neither "good" nor "bad." Still, try to be mindful about what kinds of activities you combine. For example, if a task is important and requires your attention, such as studying for an exam, it is better to make it your sole focus and avoid multitasking. In contrast, if time is 
not of the essence and there is little at stake, multitasking will not do much harm. So, while you cannot trick your brain into processing more than one task simultaneously, keeping the bottleneck theory in mind can help you multitask smartly!

\section{ACKNOWLEDGMENTS}

We thank our fellow members of the project Mechanisms and Sequential Progression of Plasticity for their insights and feedback on this article.

\section{REFERENCES}

1. Dreher, J. C., and Grafman, J. 2003. Dissociating the roles of the rostral anterior cingulate and the lateral prefrontal cortices in performing two tasks simultaneously or successively. Cereb. Cortex. 13:329-39. doi: 10.1093/cercor/ 13.4.329

2. Pashler, H. 1984. Processing stages in overlapping tasks: evidence for a central bottleneck. J. Exp. Psychol. Human. 10:358-77. doi: 10.1037/0096.1523.1 0.3 .358

3. Hoyos, P., Kim, N., and Kastner, S. 2019. How is magnetic resonance imaging used to learn about the brain? Front. Young Minds. 7:86. doi: 10.3389/frym.2019.00086

4. Worringer, B., Langner, R., Koch, I., Eickhoff, S. B., Eickhoff, C. R., and Binkofski, F. C. 2019. Common and distinct neural correlates of dual-tasking and task-switching: a meta-analytic review and a neuro-cognitive processing model of human multitasking. Brain Struct. Funct. 224:1845-69. doi: 10.1007/s00429019-01870-4

5. Dux, P. E., Tombu, M., Harrison, S., Rogers, B. P., Tong, F., and Marois, R. 2009. Training improves multitasking performance by increasing the speed of information processing in human prefrontal cortex. Neuron 63:127-38. doi: 10.1016/j.neuron.2009.06.005

6. Courage, M. L., Bakhtiar, A., Fitzpatrick, C., and Brandeau, K. 2015. Growing up multitasking: the costs and benefits for cognitive development. Dev. Rev. 35:5-41. doi: 10.1016/j.dr.2014.12.002

7. Uncapher, M. R., and Wagner, A. D. 2017. Minds and brains of media multitaskers: current findings and future directions. PNAS 115:9889-96. doi: 10.1073/pnas.1611612115

8. Karle, J. W., Watter, S., and Shedder, J. M. 2010. Task switching in video game players: benefits of selective attention but not resistance to proactive interference. Acta Psychol. 134:70-8. doi: 10.1016/j.actpsy.2009.12.007

SUBMITTED: 17 July 2020; ACCEPTED: 05 July 2021;

PUBLISHED ONLINE: 02 August 2021.

EDITED BY: Marcel Ruiz-Mejias, Pompeu Fabra University, Spain 

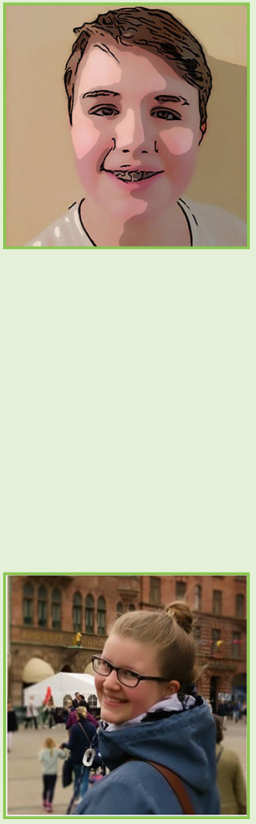

\section{AUTHORS}

\section{SINA ALEXANDRA SCHWARZE}

Sina is a neuroscience Ph.D. student fascinated by our ability to behave adequately in situations we have never been in. She is interested in how we can flexibly switch between tasks posing very different challenges to our abilities. Looking at differences in the way children and adults solve these tasks and how the activity and connections in their brains differ, she wants to learn how the brain develops to be capable of such flexible behavior. When she is not looking at images of brains, you can find her reading, cooking, doing yoga, or running and hiking in the outdoors. *schwarze ampib-berlin.mpg.de

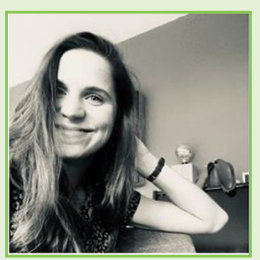

\section{CAROLINE POPPA}

Caroline is a master student, currently finishing her degree in neurobiology and behavior while helping scientists examine how plasticity changes the brain over our lifespan. She loves investigating plasticity in the human brain, using modern technologies like fMRI. Caroline is especially interested in the mechanisms underlying learning and developmental psychology. Apart from science, she is very fond of outdoor sports, arts, and indie pop music-often running through the forests of Berlin in her free time. *poppa@ampib-berlin.mpg.de 

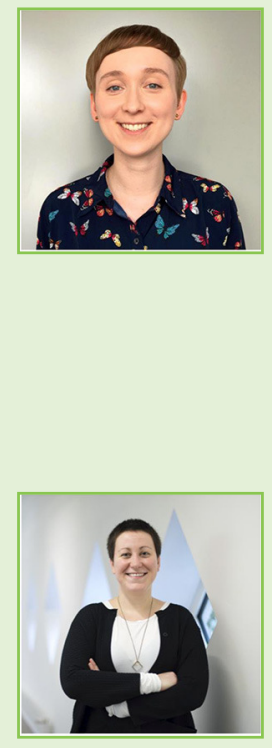

\section{SARAH MARGO GAWRONSKA}

Margo is a master student in neuroscience with aspirations to turn her passion for scientific research into her profession. While many topics in neuroscience captivate her interest, she is especially curious about the lifespan development of neural plasticity, or the ability of the brain to adapt to a changing environment Recently, her studies have focused on plasticity and rehabilitation in neurological disorders such as stroke and Parkinson's disease. Margo also enjoys honing her mandolin playing, exploring the outdoors with friends, and building extravagant cardboard forts for her cat. *gawronska@mpib-berlin.mpg.de

\section{YANA FANDAKOVA}

Yana is a developmental cognitive neuroscientist. She studies how the human brain changes as we grow up and how it helps us remember new things, achieve our goals, and accomplish many different tasks. She is also interested in how children and adults learn new skills like riding the bike or playing the piano. When she is not researching or teaching, Yana likes trying out new ice cream flavors, visiting new places, and going on long walks with her family. *fandakova@mpib-berlin.mpg.de 